\title{
PISA. The effect of paracetamol (acetaminophen) and ibuprofen on body temperature in acute stroke: Protocol for a phase II double-blind randomised placebo-controlled trial [ISRCTN98608690]
}

\author{
Eric J van Breda $\dagger^{1}$, Bart van der Worp $\dagger^{2}$, Maarten van Gemert ${ }^{3}$, \\ Ron Meijer $\dagger^{1}$, Jaap Kappelle $\dagger^{2}$, Peter J Koudstaal $\dagger^{1}$ and \\ Diederik W Dippel $\dagger^{* 1}$ for the PISA-investigators
}

Address: ${ }^{1}$ Department of Neurology, University Hospital Rotterdam, PO Box 2040, 3000 CA Rotterdam, The Netherlands, ${ }^{2}$ Department of Neurology, University Medical Centre Utrecht, PO Box 855003508 GA Utrecht, The Netherlands and ${ }^{3}$ Department of Neurology, Ziekenhuis Eemland, Ringweg Randenburg 110, 3816 CP Amersfoort, The Netherlands

E-mail: Eric Breda - breda@neuro.fgg.eur.nl; Bart van der Worp - H.B.vandeWorp@neuro.azu.nl; Maarten Gemert - vgemert@worldonline.nl; Ron Meijer - meijer@neur.azr.nl; Jaap Kappelle - l.kappelle@neuro.azu.nl; Peter J Koudstaal - koudstaal@neur.azr.nl; Diederik W Dippel* - dippel@neuro.fgg.eur.nl; the PISA-investigators - dippel@neuro.fgg.eur.nl

${ }^{*}$ Corresponding author †Equal contributors

Published: 27 March 2002

BMC Cardiovascular Disorders 2002, 2:7

This article is available from: http://www.biomedcentral.com//47I-226I/2/7

(c) 2002 Breda et al; licensee BioMed Central Ltd. Verbatim copying and redistribution of this article are permitted in any medium for any purpose, provided this notice is preserved along with the article's original URL.
Received: 29 November 2001

Accepted: 27 March 2002

\begin{abstract}
Background: During the first days after stroke, one to two fifths of the patients develop fever or subfebrile temperatures. Body temperature is a strong prognostic factor after stroke. Pharmacological reduction of temperature in patients with acute ischaemic stroke may improve their functional outcome. Previously, we studied the effect of high dose $(6 \mathrm{~g}$ daily) and low dose (3 $g$ daily) paracetamol (acetaminophen) in a randomised placebo-controlled trial of 75 patients with acute ischemic stroke. In the high-dose paracetamol group, mean body temperature at 12 and 24 hours after start of treatment was $0.4^{\circ} \mathrm{C}$ lower than in the placebo group. The effect of ibuprofen, another potent antipyretic drug, on body-core temperature in normothermic patients has not been studied.
\end{abstract}

Aim: The aim of the present trial is to study the effects of high-dose paracetamol and ibuprofen on body temperature in patients with acute ischaemic stroke, and to study the safety of these treatments.

Design: Seventy-five $(3 \times 25)$ patients with acute ischaemic stroke confined to the anterior circulation will be randomised to treatment with either: $400 \mathrm{mg}$ ibuprofen, $1000 \mathrm{mg}$ acetaminophen, or with placebo 6 times daily during 5 days. Body-temperatures will be measured with a rectal electronic thermometer at the start of treatment and after 24 hours. An infrared tympanic thermometer will be used to monitor body temperature at 2-hour intervals during the first 24 hours and at 12-hour intervals thereafter. The primary outcome measure will be rectal temperature at 24 hours after the start of treatment. The study results will be analysed on an intent-to-treat basis, but an on-treatment analysis will also be performed. No formal interim analysis will be carried out. 


\section{Background}

During the first days after stroke, between one and two fifths of the patients develop fever or subfebrile temperatures. [1-4] Increased temperatures have been associated with relatively large infarct volumes, high case fatality, and poor functional outcome, even after adjustment for initial stroke severity. [2-6] The period in which hyperthermia is associated with poor outcome is probably limited to the first 12 or 24 hours from stroke onset. [6,7]

The harmful effects of an early rise in body temperature have been attributed to increased cerebral metabolic demands, [8] changes in the blood-brain barrier permeability, acidosis, and an increased release of excitatory amino acids. [9] In animal models of temporary focal cerebral ischemia, mild intra-ischaemic hyperthermia increased infarct volume, [10] whereas mild hypothermia reduced infarct size. [11]

The above suggests that a pharmacological reduction of temperature in patients with acute ischaemic stroke may improve their functional outcome. Previously, we studied the effect on body temperature of high ( 6 g daily) and medium dose ( 3 g daily) paracetamol (acetaminophen) in a randomised placebo controlled trial of $3 \times 25$ patients (PAPAS: paracetamol (acetaminophen) in patients with acute stroke). [12] In the high dose paracetamol group, mean body temperature at 12 and 24 hours after start of treatment was $0.4^{\circ} \mathrm{C}\left(95 \%\right.$ CI: $\left.0-0.8^{\circ}\right)$ lower than in the placebo group. Koennecke conducted a comparable study of 40 patients with acute stroke. He showed that treatment with paracetamol in a daily dose of $4000 \mathrm{mg}$ resulted in a substantial reduction of the proportion of patients with body temperatures over $37.5^{\circ} \mathrm{C}$, but he did not estimate the size of the temperature reduction. [13]

Kasner et al. conducted a partly blinded trial of paracetamol against placebo in 39 patients with haemorrhagic or ischemic stroke, and oberved a difference of $0.2^{\circ} \mathrm{C}$ in body temperature. The patients who received paracetamol tended to be more often hypothermic $\left(<36.5^{\circ} \mathrm{C}\right)$ and less often hyperthermic $\left(>37.5^{\circ} \mathrm{C}\right)$, but these results were not statistically significant. [14] In this study, a lower dose (3.9 g daily) was used than in our study (6 g daily).

Several studies in adults and children with fever suggest a larger antipyretic effect from ibuprofen than from paracetamol. [15-17] The effect of ibuprofen on body-core temperature in normothermic and subfebrile patients with stroke, who may have a disturbance of thermoregulation,[18] has not been studied.

\section{Pharmacological properties and toxicity of ibuprofen}

Ibuprofen is a prostaglandin-synthetase inhibitor with analgesic, anti-inflammatory, and antipyretic features. It belongs to the group of propionic acid derivatives. It is usually well tolerated, has almost no side effects or drug interactions at therapeutic doses, and it affects the platelet aggregation in doses of more than $1 \mathrm{~g}$ by reversible inhibition of cyclo-oxygenase.

After oral administration, ibuprofen is rapidly absorbed; the maximum concentration is reached after 1 to 2 hours. Rectal administration leads to a slightly slower absorption. Ibuprofen is bound for $90 \%$ to $99 \%$ to plasma proteins, but at usual concentrations only a small part of the binding sites is occupied.

Ibuprofen is mainly distributed in the plasma compartment; the distribution volume is approximately $0.15 \mathrm{I} / \mathrm{kg}$ body weight. Elimination through the urine is almost complete within 24 hours. The metabolites do not have any detectable activity. More than $90 \%$ of a dose is found back as metabolites and their conjugates in the urine. Less than $1 \%$ is excreted as unchanged ibuprofen. The plasma half-life is 1.5 to 2.5 hours. Gastrointestinal side effects, including epigastric pain, nausea, heartburn, and sensations of fullness, are experienced by 5 to $15 \%$ of patients taking ibuprofen. The frequency of these side effects is lower than that of indomethacin or aspirin. A meta-analysis even showed that there is no significant difference in adverse effects in patients treated with ibuprofen in daily doses up to $1200 \mathrm{mg}$ compared with patients given placebo. [19] Estimates of the incidence of gastro-intestinal haemorrhage in patients with acute stroke range from $0.1 \%$ to $3 \%$. $[20,21]$ Whether treatment with non-steroidal anti-inflammatory drugs (NSAID) increases the risk of this complication in these patients is likely, but unknown.

Other side effects have been reported incidentally. These include toxic amblyopia, fluid retention, edema, skin rashes, and thrombocytopenia. Several cases of aseptic meningitis, probably caused by ibuprofen, have been reported. Allopecia has been described in negroid women who also used relaxing hair lotions and steroids simultaneously. Teratogenic effects have not been reported. [22]

\section{Pharmacological properties and toxicity of paracetamol}

Paracetamol (N-acetyl-p-aminophenol) is a para-aminophenol derivative and the major metabolite of phenacetin. It has a weak inhibitory effect on peripheral biosynthesis (which accounts for its weak anti-inflammatory activity), but it is a potent inhibitor of prostaglandin production within the central nervous system. This presumably accounts for its analgesic and antipyretic proper- 
ties. It is rapidly absorbed through the gastrointestinal tract and uniformly distributed through most body fluids. After oral administration peak plasma levels are reached after 30 min to 1 hour. [23] Rectal administration leads to a slightly slower absorption, but effective plasma levels are reached after approximately one hour. [24,25] Acetaminophen is mainly conjugated in the liver, and then excreted in the urine. Plasma half-life is 1.5 to 3 hours. Following a dose of $140 \mathrm{mg} / \mathrm{kg}$ bodyweight or more, the glucuronide pathway may become saturated, and (hepato-) toxicity may result. In patients with chronic liver failure, the threshold for toxicity is lower. [23] These patients will therefore be excluded from the present study.

\section{Methods and design}

This study is a three-armed, randomised, double-blind, placebo-controlled trial, performed in the university hospitals of Rotterdam and Utrecht, and in the regional hospital "Eemland" in Amersfoort, The Netherlands.

\section{Aim of the study}

To study the effect of high-dose ibuprofen and to confirm the previously observed reducing effect of high-dose paracetamol on body temperature, and to study their safety in normothermic and subfebrile patients with acute ischaemic stroke.

\section{Study medication}

Patients will be treated for 5 days with paracetamol or ibuprofen in daily doses of $6 \times 1000 \mathrm{mg}$ or $6 \times 400 \mathrm{mg}$, respectively, or with placebo. The study drug will be administered through identical capsules. The first gift of medication may be given as a suppository. The study medication will be provided in white paper boxes, numbered consecutively with a medication number. Each box contains 70 identical capsules containing paracetamol 500 $\mathrm{mg}$, ibuprofen $200 \mathrm{mg}$, or placebo, and one suppository, containing paracetamol $1000 \mathrm{mg}$, ibuprofen $400 \mathrm{mg}$, or placebo. Every gift of medication consists of 2 capsules except the first gift, which is one suppository. The studypopulation will be stratified according to the time since onset of stroke ( 0 to 12 hours vs 12 to 24 hours).

\section{Randomisation and treatment schedule}

Treatment allocation will be random. The randomisation will be blocked in lots of six. A computer-generated list containing the medication number and information on the nature of the treatment (placebo, paracetamol, or ibuprofen) will reside with the independent safety committee and with the study pharmacist. Treatment allocation of any patient who is in the study will be disclosed before termination of the study if the safety committee deems this necessary. Local clinical investigators may apply for disclosure of the treatment allocation of an individual patient by the study pharmacist. This will be reported to the safety committee and to the steering committee by the study pharmacist.

Pain should not be treated with NSAID. We advise codeine, 4 to 6 times $10 \mathrm{mg}$ daily, or tramadol, 4 times 25 to $50 \mathrm{mg}$ daily. When the treating physician judges that fever should be treated in a patient, the study medication should be stopped. The clinical investigators are advised to prescribe low-dose aspirin in all patients.

\section{Outcomes}

The primary outcome will be body temperature at 24 hours from start of treatment. Secondary outcomes will be change in baseline temperature at 1 and 5 days from start of treatment, and time with elevated body temperature $\left(>37.0^{\circ} \mathrm{C}\right.$ ) (area under the curve) during the first 24 hours and the first five days. The tertiary outcome is functional outcome at 1 month, as determined by the scores on the modified Rankin Scale (mRS) and Barthel Index (BI).

\section{Setting}

Ziekenhuis Eemland Amersfoort is a 600 bed regional hospital. Annually, 150 patients with acute stroke are admitted. The University Medical Centre Utrecht has 1043 beds, and admits a similar number of patients with acute ischaemic stroke. The University Hospital Rotterdam has 1229 beds. Annually, 250 patients with acute stroke are admitted. All three hospitals have an acute stroke unit and a dedicated stroke team.

\section{Study organization}

The study personnel consists of an executive committee (RJM, EJB, DWJD, HMAvG, HBvdW), an advisory committee (PJK, UK), and an independent data-monitoring and safety committee.

The co-ordinating centre for data management is located in Rotterdam. The investigators are responsible for patient selection and inclusion, and data acquisition. The investigators will notify the data management centre of newly included patients and of serious adverse events within 72 hours.

The data-management centre is responsible for prompt data entry. Every week, a progress report entailing all serious adverse events (infection, gastro-intestinal haemorrhage, liver function disturbance, neurological deterioration, death, or other) will be provided by the executive committee. These data will be combined with the randomisation scheme by the secretary of the data-monitoring and safety committee, and summarised in a simple table. This table is forwarded to the other members of the data-monitoring and safety committee. The members will acknowledge receipt of the summary table. Upon receipt of the safety data, each member of the safety committee 
will notify the advisory committee of his approval of continuation of the study. If one or more members of the safety committee express a concern with regard to the safety of the study treatment, the chairman of the safety committee will summon the members of the safety committee and try to reach a consensus or majority advise. In light of the small number of patients in the study, no formal statistical stopping rule is given.

\section{Time path}

In the previous study carried out in the same centres with similar inclusion criteria, it took 8 months to include 75 patients, i.e. 2 patients per week. It is therefore not unrealistic to take eight months for patient inclusion, one month to complete the follow-up and to close the database, and one month to report the study. The study has started in December 2000, and is expected to be completed at the end of the year 2001.

\section{Inclusion criteria}

Patients will be included if they have an acute ischaemic anterior circulation stroke, a body temperature $>36.0^{\circ} \mathrm{C}$ or $<39.0^{\circ} \mathrm{C}$, a CT scan that is compatible with acute ischaemic stroke, a focal deficit without rapid improvement, and a possibility to start treatment within $24 \mathrm{~h}$ after stroke onset.

\section{Exclusion criteria}

Criteria for exclusion from this study are: 1) severe aphasia, defined as an aphasia score of 2 or 3 on the National Institutes of Health Stroke Scale (NIHSS); 2) treatment with an NSAID deemed necessary; 3) hypersensitivity to ibuprofen or paracetamol, (chronic) liver failure or cirrhosis; 4) (chronic) renal failure; 5) history of alcohol abuse; 6) active gastric ulcer disease or a history of peptic ulceration or gastro-intestinal hemorrhage in the preceding year; 7) colitis ulcerosa; 8) pregnancy; 9) use of corticosteroids; 10) a severe concomitant medical condition that could affect the assessment of the effect of the study medication on temperature; 11 ) residual neurological impairment resulting from a previous stroke that may hamper the assessment of functional outcome; 12) death appearing imminent, and 13) no informed consent given.

\section{Study activities}

In the first 24 hours, tympanic temperature will be measured in both ears every 2 hours. During the remaining 6 days, temperature will be measured twice a day. On the first day, rectal temperature will be measured at start of treatment $(\mathrm{t}=0)$ and at $\mathrm{t}=24$ hours.

Day 0 is defined as the time period between onset of stroke and inclusion in the study. All baseline investigations are therefore carried out on day 0 . Day 1 commences directly after the start of treatment with the study medica- tion. All time-periods in the study are measured relative to the time of start of treatment.

At baseline, the medical history, including previous strokes or transient ischaemic attacks, will be assessed and a general and neurological examination will be carried out. The NIHSS is used to assess stroke severity at onset. The mRS is used to determine the patients' pre-stroke functional status. Laboratory investigations will include a full blood count, glucose, electrolytes, creatinin, AST, ALT, AF, LDH, $\gamma \mathrm{GT}$, total bilirubin, APTT and PTT, and CRP. A brain CT will be done before inclusion in the study.

Body-temperature will be measured with a tympanic thermometer[26,27] at 2-hourly intervals during the first 24 hrs after start of treatment, and thereafter every 12 hours until day 7. Temperatures will be taken from both ears, and averaged. The baseline temperature (at the start of treatment) and temperature at 24 hours after start of treatment will be determined by both rectal and tympanic thermometry. Compliance will be measured by counting leftover pills, and by a registry of the medication that was actually taken. Safety laboratory assessments will be carried out on day 6, including hemoglobin, AST, ALT, AF, $\mathrm{LDH}, \gamma \mathrm{GT}$, total bilirubin and CRP.

At the follow-up visit on day $28, \mathrm{mRS}$ is used to assess functional outcome. [28] The score on the BI will also be assessed. Based on the neurological examination and the results of CT, duplex, and other studies, etiological stroke type will be determined according to the (modified) Trial of Orgaran in Acute Stroke (TOAST) classification. [29]

\section{Safety concerns and adverse events}

One should be careful not to overlook infections in patients who are treated according to the study protocol. Therefore, treating physicians are advised to lower their thresholds for clinical suspicion of infection to $38^{\circ} \mathrm{C}$, to monitor the heart rate carefully, and start diagnostic studies and antibiotic treatment earlier than usual. The decision to start antipyretic mediciation in feverish patients is left to the discretion of the treating physician. On such an occasion, the study medication should be stopped.

Serious adverse events are defined as any deterioration in health status that is potentially life threatening, and death, within the study-monitoring period (day 0 to day 7 ). This includes any life-threatening infection, such as pneumonia, sepsis, or urinary tract infection that needs antibiotic treatment according to the judgement of the treating physician, any liver function disturbance (AST, ALT, AF, or total bilirubin levels exceeding twice the local upper limit of normal), gastro-intestinal haemorrhage, and neurological deterioration (i.e. decrease in consciousness level of more than one point on the Glasgow coma scale, or a decrease 
of two or more points on the NIHSS). All serious adverse events have to be reported within 72 hours to the independent data-monitoring and safety committee.

\section{Ethical considerations}

Each patient will be given written information about the study. Informed consent will be obtained verbally and in writing before inclusion. If the patient is unable to write, written consent will be obtained from an appropriate source (spouse, child, or parent). The medical ethics committees of the three hospitals have approved this protocol.

\section{Analysis}

The study results will be analysed on intent-to-treat basis, but an "on-treatment" analysis of primary and secondary endpoints will be carried out. There will be no formal interim analysis. The main results of the study will be presented as the mean difference in temperature between each treatment group and the placebo group. The precision of these estimates will be expressed with $95 \%$ confidence intervals, based on the $t$ distribution. No adjustments for multiple comparisons will be made. Multiple linear regression will be used to adjust for possible confounding factors, such as age, stroke type, and stroke severity. The main analysis will be carried out according to the intent to treat principle, but an on treatment analysis will be provided. The analysis will be carried out with the STATA 7.0 statistical package. [30]

\section{Sample size}

Seventy-five $(3 \times 25)$ patients will be included in the study. In a recent cohort study of 162 patients admitted within 48 hours from onset, conducted at the University Hospital Rotterdam, mean rectal temperature was $36.6^{\circ} \mathrm{C}$, with a standard deviation of $0.6^{\circ} \mathrm{C}$. In order to detect a difference in core body temperature at 24 hours from onset of $0.5^{\circ} \mathrm{C}$, with a significance level of $\alpha=0.05$, and power $1-\beta=0.80$, we need at least 23 patients in each treatment group; this is rounded to 25 . The primary outcome occurs 24 hours after start of treatment. We expect more than $90 \%$ compliance in this period. We therefore need only 2 extra patients per treatment for lost patients in the ontreatment analysis.

\section{Publication of study results}

All publications will be authored by the members of the executive committee and the advisory committee. Co-authors are supposed to have contributed to the design, the analysis and actual reporting of the study; their centres are expected to include patients roughly proportional to the number of acute stroke patients that are admitted annually.

\section{Discussion}

We report the protocol of a phase II trial that aims to assess the feasibility of early temperature reduction in normothermic or subfebrile patients with acute stroke. This study intends to confirm the previously observed temperature-reducing effect of paracetamol ( 6 g daily) and to assess the effect of ibuprofen $2.4 \mathrm{~g}$ daily.

The study is powered to find a difference of $0.5^{\circ} \mathrm{C}$ between active treatment and placebo. Half a degree Celsius does not seem much by itself, but body temperature has been shown to be a very strong prognostic factor in patients with acute stroke. For every degree Celsius of body temperature the risk of poor outcome increases by 2.2 . The earlier the increase in temperature occurs, the stronger the relationship between brain damage and high temperature seems to be. $[6,7]$

Because of the consistently observed relation between hyperthermia and poor outcome after stroke, it is commonly advised to treat all stroke patients with a raised temperature with antipyretic drugs. [31,32] However, a causal relationship between hyperthermia and poor outcome has never been demonstrated, and subfebrile temperatures and fever may just be epiphenomena of severe brain damage. In addition, there is no evidence that a pharmacological reduction of increased temperatures will lead to an improved outcome. Preventive administration of antipyretics may even mask infections and lead to delayed treatment with antibiotics. For these reasons, we think that the efficacy of antipyretic drugs to improve outcome after stroke has to be proven in a randomised controlled trial before treatment with these agents is accepted as a standard procedure. For the same reasons, we consider it ethical to withhold antipyretics from stroke patients with subfebrile temperatures in the placebo arm of such a trial.

When the effect of paracetamol or ibuprofen on body temperature in acute stroke has been established, a large, pragmatic phase III study will be undertaken to assess the effect of these cheap and well known drugs on functional outcome after stroke.

\section{The PISA investigators are}

Executive committee: EJ van Breda, DWJ Dippel, RJ Meijer, HB van de Worp, HMA van Gemert. Data-management: EJ van Breda, H Hilkemeijer, L Kasijo. Statistical analysis: DWJ Dippel. Data monitoring and safety committee: A Algra, (chairman), J van Gijn, PA van Doom, A Koudstaal-Overduin (secretary). Advisory committee: PJ Koudstaal, LJ Kappelle. Writing committee: EJ van Breda, HB van der Worp, HMA van Gemert, RJ Meijer, LJ Kappelle, PJ Koudstaal, and DWJ Dippel. Participating centers: Ziekenhuis Eemland Amersfoort, The Netherlands: 
HMA van Gemert, A Hovestadt, JBS Boringa. University Hospital Rotterdam: DWJ Dippel, MPJ van Goor, RJ Meijer, D Siepman, E Maasland, SLM Bakker, F van Kooten, A Wijnhoud. University Medical Centre Utrecht: LJ Kappelle, HB van der Worp.

\section{Competing interests}

None declared.

\section{Financial support}

The study is funded by the Stichting Neurovasculair Onderzoek Rotterdam.

\section{List of abbreviations}

NSAID - non-steroidal anti-inflammatory drug

mRS - modified Rankin Scale

BI - Barthel Index

NIHSS - National Institutes of Health Stroke Scale

AST - aspartate aminotransferase

ALT - alanine aminotransferase

AF - alkaline phosphatase

LDH - lactate dehydrogenase

$\gamma \mathrm{GT}$ - gamma glutamyl transpeptidase

APTT - activated partial thromboplastin time

PTT - prothrombin time

CRP - C-reactive protein

CT - computed tomography

TOAST - Trial of Orgaran in Acute Stroke

\section{Acknowledgements}

Special thanks to DR Paul Janssen, pharmacologist at the University Hospital Rotterdam, who prepared the study medication and gave some useful advice on the study design, and to Dr Paul Mulder (Dept of Epidemiology and Biostatistics), who provided the randomisation codes.

\section{References}

I. Przelomski MM, Roth RM, Gleckman RA, Marcus EM: Fever in the wake of a stroke. Neurology 1986, 36:427-429

2. Castillo J, Martinez F, Leira R, Prieto JM, Lema M, Noya M: Mortality and morbidity of acute cerebral infarction related to temperature and basal analytic parameters. Cerebrovasc Dis 1994, 4:66-7I

3. Azzimondi G, Bassein L, Nonino F, Fiorani L, Vignatelli L, Re G, et al: Fever in acute stroke worsens prognosis. A prospective study. Stroke 1995, 26:2040-2043
4. Hindfelt B: The prognostic significance of subfebrility and fever in ischaemic cerebral infarction. Acta Neurol Scand 1976, 53:72-79

5. Castillo J, Davalos A, Marrugat J, Noya M: Timing for fever-related brain damage in acute ischemic stroke. Stroke 1998, 29:24552460

6. Reith J, Jorgensen HS, Pedersen PM, Nakayama H, Raaschou HO Jeppesen LL, et al: Body temperature in acute stroke: relation to stroke severity, infarct size, mortality, and outcome. Lancet 1996, 347:422-425

7. Jorgensen HS, Reith J, Pedersen PM, Nakayama H, Olsen TS: Body temperature and outcome in stroke patients. Lancet 1996 , 348: 193

8. Nemoto EM, Frankel HM: Cerebral oxygenation and metabolism during progressive hyperthermia. Am J Physiol 1970, 219:1784-1788

9. Busto R, Globus MY, Dietrich WD, Martinez E, Valdes I, Ginsberg MD: Effect of mild hypothermia on ischemia-induced release of neurotransmitters and free fatty acids in rat brain. Stroke 1989, 20:904-910

10. Meden P, Overgaard K, Pedersen H, Boysen G: The influence of body temperature on infarct volume and thrombolytic therapy in a rat embolic stroke model. Brain Res 1994, 647: I3 I- I 38

II. Karibe H, Chen SF, Zarow GJ, Gafni J, Graham SH, Chan PH, et al: Mild intraischemic hypothermia suppresses consumption of endogenous antioxidants after temporary focal ischemia in rats. Brain Res 1994, 649:12-18

12. Dippel DWJ, van Breda EJ, van Gemert HM, van der Worp HB, Meijer RJ, Kappelle LJ, et al: Effect of paracetamol (acetaminophen) on body temperature in acute ischemic stroke: a double-blind, randomized phase II clinical trial. Stroke 200I, 32:1607-1612

13. Koennecke HC, Leistner S: Prophylactic antipyretic treatment with acetaminophen in acute ischemic stroke: A pilot study. Neurology 200I, 57:230I-2303

14. Kasner SE, Wein T, Piriyawat P, Villar-Cordova CE, Chalela JA, Krieger DW, et al: Acetaminophen for Altering Body Temperature in Acute Stroke: A Randomized Clinical Trial. Stroke 2002, 33:130-135

15. Kauffman RE, Sawyer LA, Scheinbaum ML: Antipyretic efficacy of ibuprofen vs acetaminophen. Am J Dis Child 1992, 146:622-625

16. Van Esch A, Van Steensel-Moll HA, Steyerberg EW, Offringa M, Habbema JD, Derksen-Lubsen G: Antipyretic efficacy of ibuprofen and acetaminophen in children with febrile seizures. Arch Pediatr Adolesc Med 1995, 149:632-637

17. Autret E, Reboul-Marty J, Henry-Launois B, Laborde C, Courcier S, Goehrs JM, et al: Evaluation of ibuprofen versus aspirin and paracetamol on efficacy and comfort in children with fever. Eur | Clin Pharmacol | 997, 5 1:367-37|

18. Mackowiak PA: Concepts of fever. Arch Intern Med 1998, I 58:1870|88|

19. Kellstein DE, Waksman JA, Furey SA, Binstok G, Cooper SA: The safety profile of nonprescription ibuprofen in multiple-dose use: a meta-analysis. / Clin Pharmacol 1999, 39:520-532

20. Wijdicks EF, Fulgham JR, Batts KP: Gastrointestinal bleeding in stroke. Stroke 1994, 25:2। 46-2।48

21. Davenport RJ, Dennis MS, Warlow CP: Gastrointestinal hemorrhage after acute stroke. Stroke 1996, 27:42 I-424

22. Mellergard $\mathrm{P}$, Nordstrom $\mathrm{CH}$ : Intracerebral temperature in neurosurgical patients. Neurosurgery |99|, 28:709-7|3

23. Clissold SP: Paracetamol and phenacetin. Drugs 1986, 32(Supp| 4):46-59

24. Blume H, Ali SL, Elze M, Kramer J, Scholz ME: The relative bioavailability of paracetamol in suppositories in comparison to tablets. Arzneimittelforschung 1996, 46:975-980

25. Kolloffel W], Driessen FG, Goldhoorn PB: Rectal administration of paracetamol: a comparison of a solution and suppositories in adult volunteers. Pharm World Sci 1996, 18:26-29

26. Postma CT, Wahjudi J, Kamps JATM, De Boo Th, Van der Meer JWM: Temperatuurmeting bij volwassenen: met de trommelvliesthermometer en de rectale digitale thermometer even goede resultaten op de afdeling Interne Geneeskunde. Ned Tijdschr Geneeskd I997, I 4 I :942-946

27. Castle SC, Toledo SD, Daskal SL, Norman DC: The equivalency of infrared tympanic membrane thermometry with standard thermometry in nursing home residents. I Am Geriatr Soc 1992, 40:1212-1216 
28. van Swieten JC, Koudstaal PJ, Visser MC, Schouten HJ, van Gijn J: Interobserver agreement for the assessment of handicap in stroke patients. Stroke 1988, 19:604-607

29. Adams HPJ, Bendixen BH, Kappelle LJ, Biller J, Love BB, Gordon DL, et al: Classification of subtype of acute ischemic stroke. Definitions for use in a multicenter clinical trial. TOAST. Trial of Org 10172 in Acute Stroke Treatment. Stroke 1993, 24:35-4 I

30. Stata Corporation: STATA Statistics/data analysis. [7.0]. College Station, Texas, USA. Ref Type: Computer Program 2001

31. De Keyser J: Antipyretics in acute ischaemic stroke. Lancet 1998, 352:6-7

32. McDJohnston A: Antipyretic therapy is important following stroke. Arch Intern Med 2000, 160:2679-2680

\section{Pre-publication history}

The pre-publication history for this paper can be accessed here:

http://www.biomedcentral.com/1471-2261/2/7/prepub

Publish with BioMed Central and every scientist can read your work free of charge

"BioMedcentral will be the most significant development for disseminating the results of biomedical research in our lifetime." Paul Nurse, Director-General, Imperial Cancer Research Fund

Publish with BMC and your research papers will be:

- available free of charge to the entire biomedical community

- peer reviewed and published immediately upon acceptance

- cited in PubMed and archived on PubMed Central

- yours - you keep the copyright
BioMedcentral.com editorial@biomedcentral.com 